\title{
EFEITO HIPOGLICÊMICO DE RAÇÕES À BASE DE BERINJELA (Solanum melongena,L.) EM RATOS ${ }^{1}$
}

\author{
Sandra Casa Nova DERIVI ${ }^{2, *}$, Maria Heidi Marques MENDEZ ${ }^{2}$, Alessandro Dinuci FRANCISCONI ${ }^{3}$, \\ Cristiane Santos da SILVA ${ }^{3}$, Amanda Ferreira de CASTRO ${ }^{3}$, Daniel Percego LUZ ${ }^{3}$
}

\begin{abstract}
RESUMO
Os objetivos do presente trabalho foram verificar se dietas ricas em berinjela apresentam efeito hipoglicêmico, se contribuem para o retardamento de absorção da glicose pós-prandial e se a fração fibra solúvel está correlacionada com estes efeitos. Os experimentos foram realizados com ratos Wistar machos adultos, normais e diabéticos, que receberam rações à base de caseina, berinjela com casca e sem casca, e de casca de berinjela por um periodo de 42 dias. A glicose sangüinea foi determinada no início do experimento e aos 13 , 21,34 e 42 dias. Os testes orais de tolerância à glicose (TOTG) foram realizados no final do experimento. Os resultados mostraram que as rações à base de farinha de berinjela com casca e de casca de berinjela apresentaram redução nos niveis de glicose. Este efeito não foi significativo para a ração à base de farinha de berinjela sem casca. Os animais do grupo diabético que receberam ração à base de berinjela com casca apresentaram menor área sob a curva de glicose, do que os dos grupos controle (ração à base de caseína e de berinjela com casca) e do que o do grupo diabético que recebeu a ração de caseína. Este efeito não foi observado nos animais que receberam ração à base de berinjela sem casca e casca de berinjela. Estes resultados indicam a presença de um composto responsável pelo efeito hipoglicêmico na casca de berinjela e que a pectina solúvel da ração não foi suficiente para promover tal efeito, mas que a administração continua de pectina solúvel contribuiu para a melhora da TOTG.
\end{abstract}

Palavras-chave: diabetes; efeito hipoglicêmico; pectina solúvel; berinjela.

\section{SUMMARY}

HYPOGLYCEMIC EFFECT OF EGGPLANT (Solanum melongena, L.) IN RATS. The objectives of this work were to verify if eggplant diets exhibit a hypoglycemic effect, if these diets contribute to the post-prandial delay of glucose absorption and if the soluble fiber fraction (pectin) correlates with these effects. Male, adult Wistar rats, both normal and diabetic (alloxan induced) were fed diets based on casein, eggplant with and without the peel and eggplant peel for a 42 days. Blood glucose was determined at the onset of the experiment and at 13, 21, 34 and 42 days. Glucose tolerance oral tests were performed at the end of the experiment. Diets based on eggplant with peel flour and eggplant peel flour showed a significant reduction of plasma glucose levels, but this effect was not observed in the peeled eggplant diet. Diabetic animals consuming the diet based on eggplant with peel exhibited a lesser area under the glucose curve than the other control groups receiving the diets based on casein and eggplant-with-peel and the casein control group. In the animals that received the diets based on peeled eggplant and the eggplant peel, this effect was not observed. It is concluded that the compound responsible by the hypoglycemic effect is present in the peel of the eggplant. It is also concluded that soluble pectin in the diets was not in itself sufficient to promote this effect, but that the continuous administration of the soluble fiber contributed to the improvement of the glucose tolerance.

Keywords: diabetes; hypoglycemic effect; soluble pectin; eggplant.

\section{1 - INTRODUÇÃO}

A procura na medicina popular de fontes naturais para prevenção e tratamento das doenças da civilização, entre elas a diabetes, vem sendo cada vez mais intensificada. A importância da inclusão de alimentos que proporcionam uma melhora na tolerância à glicose, em dietas de indivíduos diabéticos, tem sido enfatizada. Atualmente, o extrato e o suco de berinjela, têm sido utilizados para diminuir as taxas de colesterol e de LDL colesterol [10]. No entanto estudos com a berinjela cozida, como é consumida em preparações alimentícias, não são descritos na literatura.

Dietas com alto teor de fibra alimentar têm apresentado resultados positivos em relação a tolerância à glicose, redução de hiperglicemia pós-prandial e taxa secretória de insulina, em indivíduos diabéticos. A fração da fibra solúvel é apontada como responsável por estes efeitos fisiológicos benéficos e vários mecanismos têm sido propostos para explicar sua ação:
Alteração na velocidade de difusão da glicose, devido à formação de gel no lúmem intestinal [6, 15, 17, 21]. Alteração na estrutura da mucosa intestinal, com rarefação das criptas e vilosidades da mucosa intestinal e aumento da produção de mucina, que atua como uma barreira à absorção de glicose[3, 4, 8, 19]. A produção de ácidos graxos de cadeia curta, como o acetato e o propionato, em decorrência da fermentação da fibra solúvel pelas bactérias do cólon, também exercem efeitos na diminuição das taxas de glicose e colesterol sangüíneo. $\mathrm{O}$ acetato inibe a lipólise do tecido adiposo que é responsável pelo excesso de ácidos graxos livres que chega ao figado e acarreta a produção de acetoacetato em indivíduos diabéticos [16, 23].

O presente trabalho tem como objetivo verificar se dietas ricas em berinjela apresentam efeito hipoglicêmico e uma melhora a tolerância à glicose, e se a presença de fibra solúvel (pectina solúvel) é um fator determinante nestes efeitos.

\footnotetext{
${ }^{1}$ Recebido para publicação em 06/07/01. Aceito para publicação em 05/02/02.

${ }^{2}$ Universidade Federal Fluminense - Departamento de Bromatologia. Rua Mário Viana, 523, Santa Rosa, CEP 24241-000, Niterói, RJ. E-mail: mboscnd@vm.uff.br

${ }^{3}$ Iniciação Científica $\mathrm{CNPq} / \mathrm{PIBIC/UFF}$

* A quem a correspondência deve ser enviada.
} 


\section{2 - MATERIAL E MÉTODOS}

\section{1 - Material}

\subsection{1 - Rações experimentais}

Foram oferecidas aos animais rações de caseína, de berinjela com e sem casca, e de casca de berinjela. A berinjela foi adquirida no comércio local. Um lote de berinjelas foi lavado e cortado em tiras e outro lote foi lavado, retirado a casca, e ambos os lotes foram cozidos por 10 minutos em água fervente. A água de cozimento foi separada para ser incorporada à ração. As cascas de berinjela foram cozidas em microondas por 3 minutos. As berinjelas, com casca e sem casca, e as cascas de berinjelas foram secas em estufa ventilada à temperatura de $55^{\circ} \mathrm{C}$, durante 24 horas, e o material foi moído e passado em tamis de 28 mesh para a obtenção das farinhas usadas no preparo das rações.

As rações seguem as formulações apresentadas na Tabela 1.

TABELA 1. Formulação das rações (g/100g de ração)

\begin{tabular}{lcccc}
\hline \multirow{2}{*}{ COMPONENTES } & \multicolumn{4}{c}{ RAÇÃO } \\
\cline { 2 - 5 } & Caseína & $\begin{array}{c}\text { Berinjela com } \\
\text { casca }\end{array}$ & $\begin{array}{c}\text { Berinjela sem } \\
\text { casca }\end{array}$ & $\begin{array}{c}\text { Casca de } \\
\text { berinjela }\end{array}$ \\
\hline Caseína & 12,5 & 12,5 & 12,5 & 12,5 \\
Berinjela com casca & - & 50,0 & - & - \\
Berinjela sem casca & - & & 50,0 & - \\
Casca de berinjela & - & - & - & 15,0 \\
Óleo de soja & 8,0 & 8,0 & 8,0 & 8,0 \\
Celulose & 4,0 & 4,0 & 4,0 & 4,0 \\
Mistura salina & 4,0 & 4,0 & 4,0 & 4,0 \\
Mistura vitamínica & 1,0 & 1,0 & 1,0 & 1,0 \\
Amido de milho & 70,5 & 20,5 & 20,5 & 55,5 \\
\hline
\end{tabular}

A mistura salina empregada segue as recomendações de FOX \& BRIGGS [7] e a mistura vitamínica, as especificações de LAJOLO et al. [11].

\subsection{2 - Animais de experimentação}

Foram utilizados um total de 106 ratos machos, adultos, da linhagem "Wistar", procedentes do Biotério do Laboratório de Nutrição Experimental, da Faculdade de Nutrição da UFF, com peso médio de 200 g. Durante o período experimental, a temperatura do biotério foi mantida a $21 \pm 2{ }^{\circ} \mathrm{C}$, com alternância automática, em períodos de 12 horas, de claro-escuro. Os animais receberam ração e água ad libitum.

A indução da diabetes foi feita com solução salina de aloxana $(40 \mathrm{mg} / \mathrm{Kg}$ de peso corporal) via veia do pênis, após um período de 24 horas de jejum, em 76 ratos. Foram considerados, para a formação dos grupos diabéticos, os ratos que apresentaram melhor resposta após 5 dias da indução. De acordo com TONIOLO et al. [20] são considerados diabéticos, os animais que após a indução com aloxana apresentarem um nivel de glicose sangüínea igual ou maior que a média do grupo controle acrescentado de 3 vezes o desvio padrão deste grupo.
Foi determinada a taxa de glicose sangüínea nos ratos normais e diabéticos (tempo $\mathrm{T}_{0}$ ), e os ratos foram divididos, em grupos homogêneos, que receberam as seguintes rações:

Grupo 1 (G 1) - Grupo Controle - Ração de caseína

Grupo 2 (G 2) - Grupo Diabético - Ração de caseína

Grupo 3 (G 3) - Grupo Controle - Ração de berinjela com casca Grupo 4 (G 4) - Grupo Diabético - Ração de berinjela com casca Grupo 5 (G 5) - Grupo Controle - Ração de berinjela sem casca Grupo 6 (G 6) - Grupo Diabético - Ração de berinjela sem casca Grupo 7 (G 7) - Grupo Controle - Ração de casca de berinjela Grupo 8(G 8) - Grupo Diabético - Ração de casca de berinjela

Foi determinada a glicose sangüínea nos tempos de: $\mathrm{T}_{1}$ (13 dias); $\mathrm{T}_{2}$ (21dias); $\mathrm{T}_{3}$ (34 dias) e $\mathrm{T}_{4}$ (42 dias).

\section{2 - Métodos}

\subsection{1 - Composição centesimal}

A umidade foi determinada gravimetricamente, por perda de peso, em estufa a $105^{\circ} \mathrm{C}$ até peso constante; o extrato etéreo foi obtido por extração contínua com éter etílico, em aparelho de Soxhlet, e a cinza foi obtida por incineração do material em mufla a $550^{\circ} \mathrm{C}$, de acordo com os métodos descritos pelo INSTITUTO ADOLFO LUTZ [9]. O nitrogênio total foi determinado pelo método de microkjeldahl e para expressar o resultado em proteína foram usados os fatores de 6,25 para a berinjela e 6,38 para a caseína[1]. A fibra detergente ácido (ADF) foi determinada pelo método de VAN SOEST [22] e a fibra detergente neutro foi determinada pelo método de MENDEZ et al. [13]. A lignina foi separada do resíduo de ADF, por meio de ácido sulfúrico a $72 \%$ e determinada gravimetricamente. A celulose foi calculada por diferença entre os valores obtidos para o resíduo de ADF e lignina. As hemiceluloses foram calculadas por diferença entre os valores obtidos para NDF e ADF. A pectina solúvel foi extraída de acordo com a técnica descrita por MCCREADY \& MCCOMB [12] e o doseamento foi feito pelo método colorimétrico segundo técnica descrita por BITTER \& MUIR [2].

\subsection{2 - Consumo de ração}

Os animais foram alojados em gaiolas metabólicas individuais e alimentados com cerca de $25 \mathrm{~g} /$ dia das rações experimentais. O consumo de ração foi anotado diariamente sendo calculado sobre as sobras verificadas no dia seguinte.

\subsection{3 - Determinação da glicose}

A glicose sangüinea foi determinada utilizando o método da hexoquinase, com o uso de fitas glicotestes (tiras reagentes Glucotide), e a leitura feita no aparelho Glicometer. 


\subsection{4 - Teste oral de tolerância à glicose}

Após um período experimental de 42 dias, os animais do grupo controle (ração de caseína) e dos grupos experimentais (ração de berinjela, com e sem casca, e ração de casca de berinjela), normais e diabéticos, foram colocados em jejum por um periodo de 12 horas. Foi recolhida uma amostra de sangue, correspondendo ao T0 (Tempo zero). A seguir foi administrada, através de sonda intragástrica, uma dose de glicose correspondente a $1 \mathrm{~g} / \mathrm{Kg}$ de peso corporal. Amostras de sangue para determinação da glicose foram colhidas nos tempos T1 (30min), T2 (60 min), T3 (120 min) e T4 (180 $\min )$.

\subsection{5 - Análise estatística}

Os dados foram analisados utilizando o programa Grahpad Prism versão 2.0 para obtenção de médias, para o cálculo da área sob a curva de glicose (mg/100mL/ min) e o teste de t de Student para comparação entre médias de dois grupos [14].

\section{3 - RESULTADOS E DISCUSSÃO}

Os teores de pectina solúvel e os valores de fibra total insolúvel são apresentados na Tabela 1.

TABELA 1. Teores de fibra da dieta insolúvel e solúvel das rações experimentais

\begin{tabular}{lcccc}
\hline \multicolumn{1}{c}{ Determinações } & \multicolumn{4}{c}{ RAÇÕES } \\
\cline { 2 - 5 } & Caseína & $\begin{array}{c}\text { Berinjela com } \\
\text { casca }\end{array}$ & $\begin{array}{c}\text { Berinjela sem } \\
\text { casca }\end{array}$ & $\begin{array}{c}\text { Casca de } \\
\text { Berinjela }\end{array}$ \\
\hline $\begin{array}{l}\text { Fibra da dieta } \\
\text { insolúvel }\end{array}$ & 4,31 & 20,99 & 18,54 & 14,42 \\
Celulose & 3,91 & 8,62 & 11,18 & 4,33 \\
Hemiceluloses & 0,37 & 0,42 & 3,19 & 0,96 \\
Lignina & 0,03 & 11,95 & 4,17 & 9,13 \\
Fibra da dieta & Zero & 1,01 & 0,86 & 0,37 \\
solúvel & & & 0,86 & 0,37 \\
Pectina solúvel & Zero & 1,01 & & \\
\hline
\end{tabular}

Resultados expressos em g/100g de material

Experimentos realizados têm apontado a presença de pectina solúvel em alimentos como responsável pelo efeito hipoglicêmico em animais diabéticos. Estudo realizado por DERIVI et al. [5] mostrou que ratos diabéticos apresentavam redução dos níveis de glicose sangüínea, quando alimentados com rações contendo $2 \%$ e $4 \%$ de pectina solúvel. Em experimento realizado em um periodo de 42 dias com ratos diabéticos que receberam uma ração formulada com $20 \%$ de farinha de jiló, adicionada da água de cozimento, (com um teor de $1,80 \mathrm{~g} \%$ de pectina solúvel e equivalente a $100 \mathrm{~g}$ de jiló cozido) foi observada uma tendência acentuada na redução dos niveis de glicose sangüinea [18]. FREITAS et al. [8] verificaram, em ratos diabéticos que receberam ração à base de sopa de cebola adicionada de pectina (contendo $2,91 \%$ de pectina solúvel), por um período de 42 dias, uma redução nos niveis de glicose sangüínea. No presente estudo, os testes foram realizados com rações contendo teores de pectina solúvel de 1,01\% (ração de berinjela com casca),
0,86\% (ração de berinjela sem casca) e 0,37\% (ração de casca de berinjela).

Na Tabela 2 são apresentados o consumo alimentar (em g), peso inicial, peso final e o ganho de peso (em g) dos ratos, normais e diabéticos, submetidos à ração de caseína e as rações experimentais de berinjela. O consumo de ração não apresentou diferença entre os grupos testados (Tabela 2), no entanto foi observado um ganho de peso menor nos ratos alimentados com ração de berinjela com casca.

TABELA 2. Peso corporal, ganho de peso e consumo alimentar (em g), dos animais submetidos à dieta de caseína, berinjela com casca e sem casca e casca de berinjela.

\begin{tabular}{|c|c|c|c|c|c|c|c|c|}
\hline Grupos/Ração & $\begin{array}{c}\mathrm{Gl} \\
\text { Controle } \\
\text { Caseina } \\
\mathrm{N}=12\end{array}$ & $\begin{array}{c}\text { G2 } \\
\text { Diabético } \\
\text { Caséina }\end{array}$ & $\begin{array}{c}\mathrm{G} 3 \\
\text { Controle } \\
\text { berinjela } \\
\text { c/casca } \\
\mathrm{N}=6\end{array}$ & $\begin{array}{c}\mathrm{G} 4 \\
\text { Diabético } \\
\text { berinjela } \\
\mathrm{c} / \text { casca } \\
\mathrm{N}=6\end{array}$ & $\begin{array}{c}\mathrm{G} 5 \\
\text { Controle } \\
\text { berinjela } \\
\mathrm{s} / \text { casca } \\
\mathrm{N}=6\end{array}$ & $\begin{array}{c}\mathrm{G} 6 \\
\text { Diabético } \\
\text { berinjela } \\
\mathrm{s} / \text { casca } \\
\mathrm{N}=6\end{array}$ & $\begin{array}{c}\mathrm{G} 7 \\
\text { Controle } \\
\text { casca de } \\
\text { berinjela } \\
\mathrm{N}=6\end{array}$ & $\begin{array}{c}\mathrm{G} 8 \\
\text { Diabético } \\
\text { casca de } \\
\text { berinjela } \\
\mathrm{N}=6\end{array}$ \\
\hline Peso inicial & 246,9 & 235,0 & 271,0 & 235,0 & 243,0 & 219,0 & 235,2 & 217,2 \\
\hline Peso final & 341,8 & 330,8 & 309,0 & 289,3 & 332,7 & 296,8 & 325,5 & 297,6 \\
\hline Ganho de peso & 94,9 & 95,8 & 38,0 & 54,43 & 89,7 & 77,8 & 90,3 & 80,4 \\
\hline $\begin{array}{l}\text { Consumo total de } \\
\text { ração }\end{array}$ & 671,5 & 681,8 & 714,3 & 712,9 & 791,5 & 740,4 & 717,25 & 743,6 \\
\hline
\end{tabular}

As Figuras 1, 2 e 3 representam as curvas de glicose versus tempo. Os experimentos com ratos diabéticos que receberam rações contendo berinjela com casca $(1,01 \mathrm{~g} \%$ de pectina solúvel), berinjela sem casca $(0,86 \mathrm{~g} \%$ de pectina solúvel) e casca de berinjela $(0,37 \mathrm{~g} \%$ de pectina solúvel) mostraram que a ração de berinjela com casca (Figura 1) e de casca de berinjela (Figura 3) apresentaram efeito hipoglicêmico, nos ratos diabéticos. A ração de berinjela sem casca (Figura 2) mostrou uma redução nos níveis de glicose sangüínea, não atingindo, no entanto os niveis basais, como verificado com as rações de berinjela com casca e de casca de berinjela. Portanto, foi evidenciado que rações contendo berinjela com casca e casca de berinjela, mesmo em presença de baixos teores de pectina solúvel, apresentaram acentuado efeito hipoglicêmico, enquanto que os teores de $0,86 \mathrm{~g} \%$ de pectina solúvel presente na ração de berinjela sem casca promoveram um efeito menos acentuado. Estes resultados apontam a presença de um outro fator contribuindo para este efeito hipoglicêmico, possivelmente a presença de um componente termo estável na casca da berinjela, apontando como mecanismo de ação a hipótese de estimular a liberação de insulina e favorecer a captação de glicose pelas células insulino dependentes dos tecidos.

Ratos diabéticos alimentados por um período de 42 dias com dietas experimentais de berinjela (berinjela com casca e sem casca e casca de berinjela), apresentaram comportamentos diferentes para o TOTG, como pode ser observado nas Figuras 5, 6 e 7. Estes resultados mostraram que ratos diabéticos que receberam dietas contendo berinjela com casca (teores de $1,01 \mathrm{~g} \%$ de pectina solúvel) apresentaram uma resposta ao TOTG que não apresentou diferença significativa em relação aos ratos normais que receberam a mesma ração (Figura 5). Os 
ratos diabéticos que receberam rações contendo berinjela sem casca (teores de $0,86 \mathrm{~g} \%$ de pectina solúvel) e casca de berinjela (teores de $0,37 \mathrm{~g} \%$ de pectina solúvel) após 3 horas da administração de glicose não alcança-

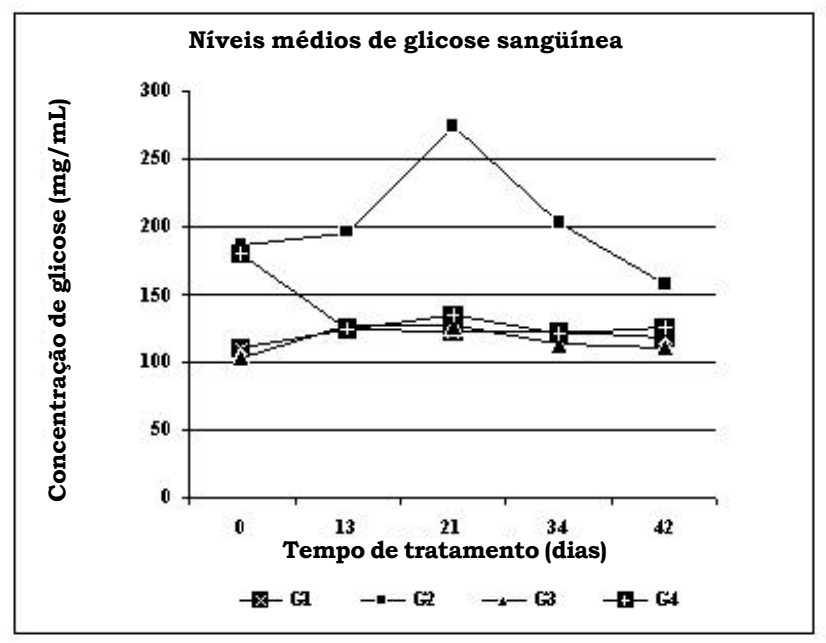

$\mathrm{G} 1$ * diferença significativa em relação a $\mathrm{G} 2(\mathrm{p}<0,05)$

G3 nã apresenta diferença significativa em relação a G4 $(\mathrm{p}<0,05)$

FIGURA 1. Niveis médios de glicose sangüinea $(\mathrm{mg} / \mathrm{mL})$ nos tempos de tratamento (dias) de ratos normais e diabéticos que receberam ração de caseína de berinjela com casca.

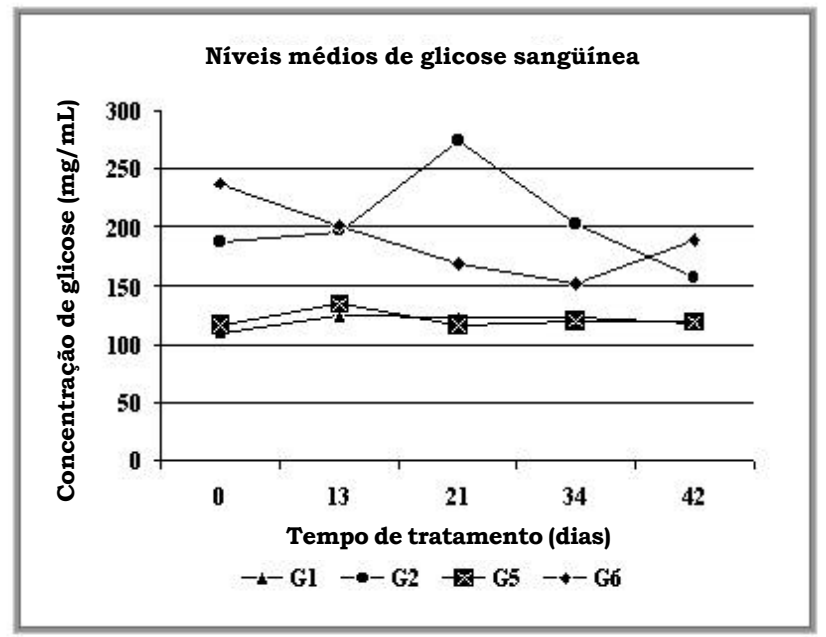

G1* diferença significativa em relação a $\mathrm{G} 2(\mathrm{p}<0,05)$

$\mathrm{G} 5$ * apresenta diferença significativa em relação a G6 $(\mathrm{p}<0,05)$

FIGURA 2. Níveis médios de glicose sangüinea $(\mathrm{mg} / \mathrm{mL})$ nos tempos de tratamento (dias) de ratos normais e diabéticos que receberam ração de caseína de berinjela sem casca.

O teste oral de tolerância à glicose (TOTG) tem sido utilizado para verificar o comportamento dos alimentos em relação ao metabolismo de açúcares. A elevação da glicemia pós-prandial, após uma sobrecarga de glicose, e a conseqüente normalização aos niveis basais, após cerca de 2 horas, caracteriza uma função normal do metabolismo glicídico. Na Figura 4 é apresentada a área sob a curva de glicose, dos ratos normais e diabéticos, alimentados com ração de caseína, usada como controle no experimento. ram os niveis basais de glicose sangüinea (Figuras $6 e$ 7), apresentando uma resposta ao TOTG diferentemente significativa em relação aos ratos normais que receberam as mesmas rações.

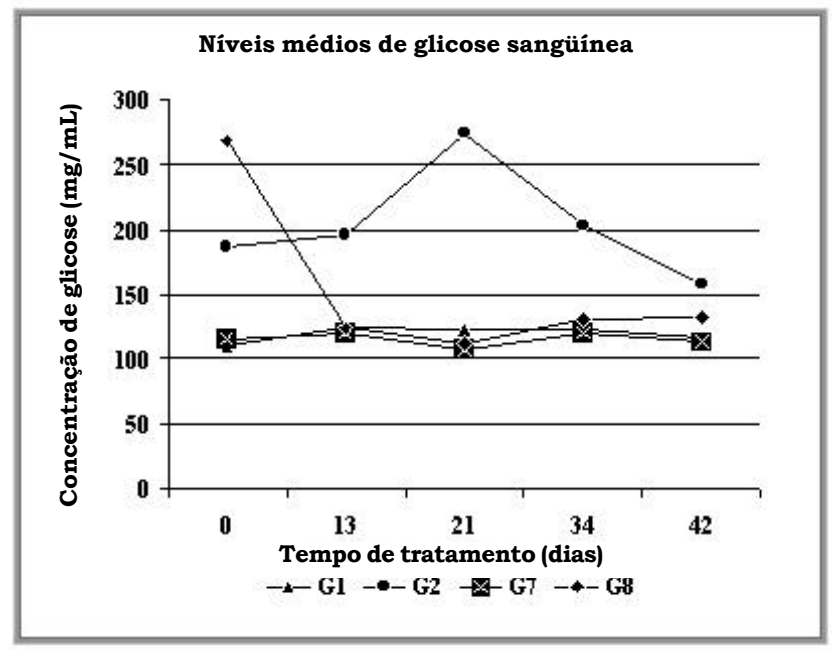

$\mathrm{G} 1$ * diferença significativa em relação a $\mathrm{G} 2(\mathrm{p}<0,05)$

G7 não apresenta diferença significativa em relação a G8 $(\mathrm{p}<0,05)$

FIGURA 3. Níveis médios de glicose sangüínea $(\mathrm{mg} / \mathrm{mL})$ nos tempos de tratamento (dias) de ratos normais e diabéticos que receberam ração de caseína de casca de berinjela.

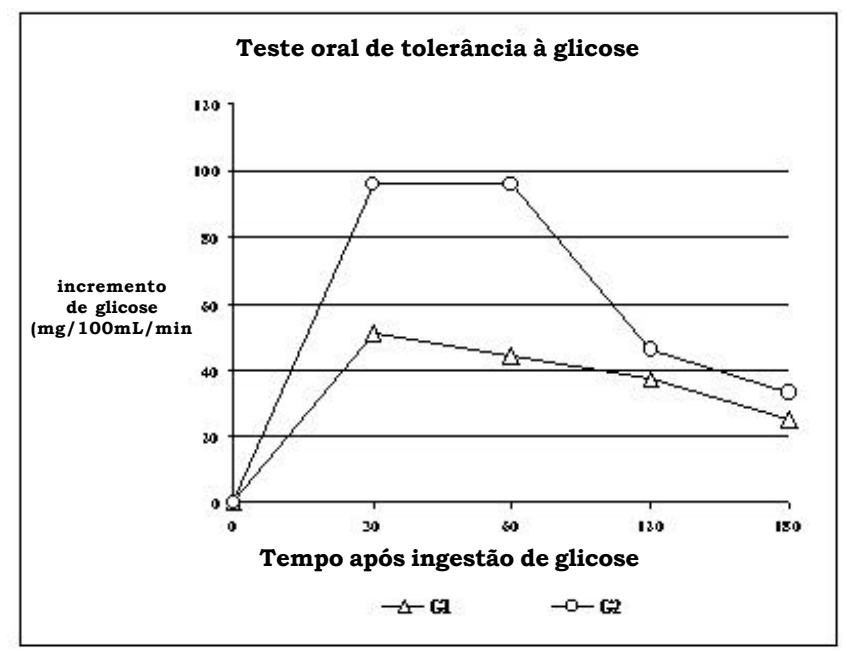

G1 - A $=23165$

$\mathrm{G} 2-\mathrm{A}=31085$

G2 *** diferença significativa em relação a G1 $(\mathrm{p}<0,0001)$

FIGURA 4. Teste oral de toelrância à glicose de ratos normais e diabéticos que receberam ração de caseína.

A pectina solúvel é responsável por alterações na estrutura da mucosa intestinal, com aparecimento de rarefação das criptas e vilosidades da mucosa intestinal [8], que diminuem a absorção de glicose. A ingestão por um período prolongado de pectina solúvel (teores de $1,01 \mathrm{~g} \%)$, presente na ração de berinjela com casca pode ser responsável pela melhora observada em relação a tolerância à glicose, em conseqüência destas alterações. 


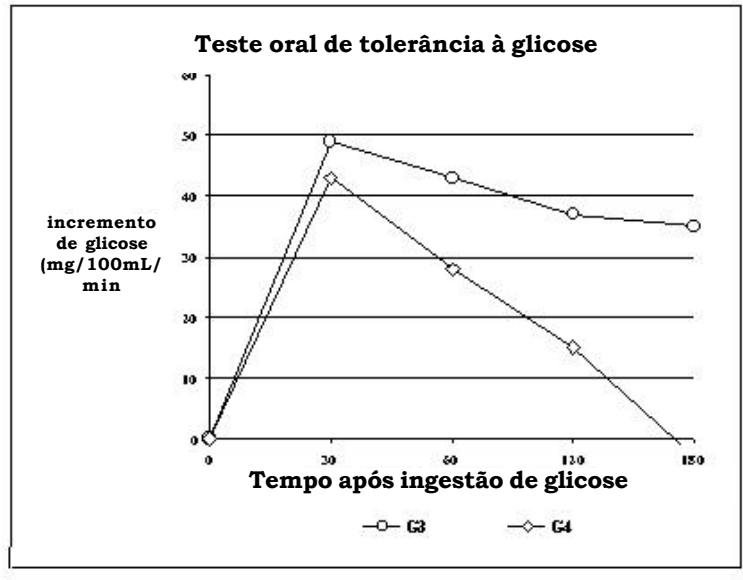

G3 - A $=22460$

$\mathrm{G} 4-\mathrm{A}=23390$

G3 não diferença significativa em relação a G4

FIGURA 5. Teste oral de toelrância à glicose de ratos normais e diabéticos que receberam ração de berinjela com casca.

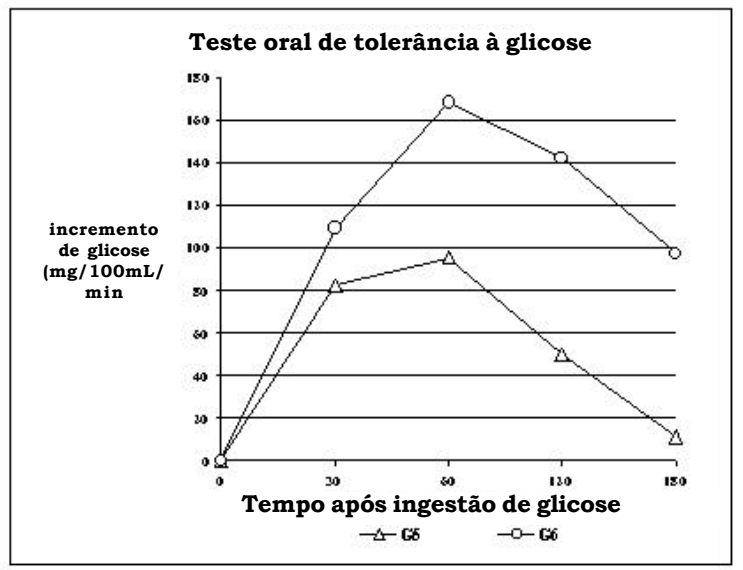

G5 - A $=28370$

$\mathrm{G} 6$
$\mathrm{G} 5$
$* * * *$

FIGURA 6. Teste oral de toelrância à glicose de ratos normais e diabéticos que receberam ração de berinjela sem casca.

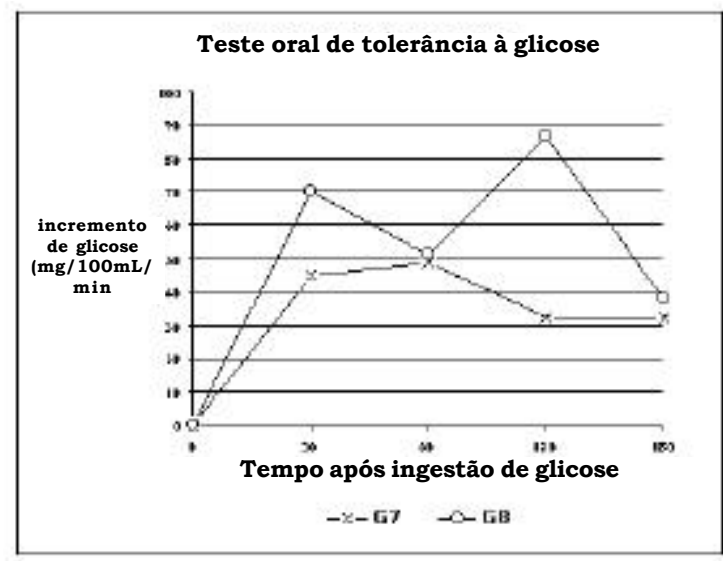

$\mathrm{G} 7-\mathrm{A}=25060$

$\mathrm{G} 8-\mathrm{A}=34530$

$\mathrm{G} 7$ *** diferença significativa em relação a $\mathrm{G} 8(\mathrm{p}<0,05)$

FIGURA 7. Teste oral de toelrância à glicose de ratos normais e diabéticos que receberam ração de casca de berinjela.

\section{4 - CONCLUSÕES}

A casca de berinjela poderá conter um componente termo estável com ação hipoglicêmica e que a ingestão continuada de teores de $1,01 \%$ de pectina solúvel contribuiu para a melhora da tolerância à glicose.

\section{5 - REFERÊNCIAS BIBLIOGRÁFICAS}

[1] ASSOCIATION OF OFFICIAL ANALYTICAL CHEMISTS. Official methods of analysis. 14 ed. Virginia, 1984, p. 988.

[2] BITTER,T.; MUIR, H.M.A Modified uronic acid carbazole reaction. Analytical Biochemistry,v.4, p. 330-334, 1962.

[3] BROWN, E.C.; KELLEHER, J.; LOSOWSKY, M.S. The effect of pectin on the structure and function of the rat small intestine. British Journal Nutrition, v. 42, n. 3, p. 357 365, 1979.

[4] CASSIDY, M.M.; LIGHFOOT, F.G.; GRAU, L.E.; STORY, J.A.; KRITCHEVSKY, D.; VAHOUNY, G.V. Effect of chronic intake of dietary fibers on the ultraestrutural topograpy of rat jejunum and colon: a scanning electron microscopy study. American Journal Clinical Nutrition, v. 34, n. 2, p. 218-228, 1981.

[5] DERIVI, S.C.N.; MENDEZ, M.H.M.; RODRIGUES, M.C.R.; FERNANDES, M.L.; SILVA, M.F. Ação da fibra solúvel pectina - sobre os niveis glicêmicos, em ratos. Revista Brasileira de Farmácia, v. 68, p. 1-7, 1987.

[6] EBIHARA, K.; KIRIYAMA, S. Comparative effects of watersoluble and water-insoluble dietary fibers on various paramenters relating to glucose tolerance in rats. Nutrition Reports International, v. 226, n. 22, p. 139202, 1982.

[7] FOX, M.R.S.; BRIGGS, G.M. Salt mixtures type diets. III Na improvised salt misture for chicks. Journal of Nutrition,v.72, p. 242-250, 1960.

[8] FREITAS, M.C.J.; DERIVI, S.C.N.; MENDEZ, M.H.M.; FERNANDES, M.L. Produto rico em fibra solúvel (pectinas) e seu efeito sobre os níveis de glicose no soro sangüíneo. Ciência e Tecnologia de Alimentos, v. 14, n. 12 , p. 46-54, 1994.

[9] INSTITUTO ADOLF LUTZ. São Paulo. Normas Analiticas do Instituto Adolf Lutz.3ed., São Paulo, 1985, v. 1, p. 21$27,42$.

[10] JORGE, P. A.R.; NEYRA, L.C.; OSAKI, R.M.; ALMEIDA, E.; BROGAGNOLO, N. Efeito da berinjela sobre os lípides plasmáticos, a peroxidação lipídica e a reversão da disfunção endotelial na hipercolesterolemia experimental. Arquivos Brasileiros de Cardiologia, v. 70, n. 2, p. 87-91, 1998.

[11] LAJOLO, F.M.; CAMPOS FRANÇA M.H.; ZUCAS, S.M. Influência da cor da ração no consumo da mesma por ratos (Rattus norvegicus, var. albinus). Revista da Faculdade de_Farmácia e Bioquimica da USP, v. 7, p. 95103, 1969.

[12] McCREADY, R.M.; McCOMB, E.A. Extration and determination of total pectin material in fruits. Analytical Chemistry, v. 24, n. 12, p. 1986-1988, 1952.

[13] MENDEZ, M.H.M.; DERIVI, S.C.N.; RODRIGUES, M.C.R.; FERNANDES, M. L.;MACHADO, R.L.D. Método de fibra detergente neutro modificado para amostras ricas em amido. Ciênc. e Tecnol. Aliment., v. 5, n. 2, p. 123$131,1985$.

[14] MOTULSKY. Intuitive Bioestatistics. New York, Oxford University Press, 1995. 
[15] RAY, T. K.; MANSELL, K.M.; KNIGHT, L.C.; MALMUD, L.S.; OWEN, E.; BODEN, G. Long-term effects of dietary fiber on glucose tolerance and gastric emptying in non insulindependent diabetic patients. American Journal of Clinical Nutrition, v. 53, p. 376-381, 1983.

[16] ROMBEAU,J.L. \& ROTH, J.A. Short-chain fatly acids research and clinical up dates. In: KRITCHEVSKY,D.; BONFIELD, C. (Ed.) Dietary fiber in health \& disease. Saint Paul: Eagan Press, 1995, p. 441-449.

[17] SCHWARTZ, S.; LEVINE, R.A; WEINSTOCK, R. S.; PETOKAS, S.; MILLS, C.A.; THOMAS, F.D. Sustained pectin ingestion: effect on gastric and glucose tolerance in non insulin- dependent diabetic patients. American Journal of Clinical Nutrition, v. 48, n. 6, p. 1413-1417, 1988.

[18] SILVA, M.B.; MENDEZ, M.H.M.; DERIVI, S.C.N. Efeito hipoglicêmico de alimentos ricos em fibra solúvel. Estudo com o jiló (Solanum gilo, Raddi). Alimentos e Nutrição, v. 9, p. 43-64, 1998.
[19] TASMAN-JONES, C.; FRACP,F.; OWEN, R.I.; JONES, A. J. Semipurified dietary fiber and small bowel morphology in rats. Digestive Diseases and Sciences, v. 27, n. 6, p. 519-529, 1982.

[20] TONIOLO, A.; ONDERA, T.; YOON, J.W.; NOTKINS, AL. Induction of diabetes by cumulative enviromental insults from viruses and chemical. Nature, v. 288, p. 383-385, 1980.

[21] VAHOUNY, G.V. Conclusions and recommendations of the symposium on Dietary fibers in health and disease. American Journal of Clininical Nutrition, v. 35, p. 152-156, 1982.

[22] VAN SOEST, P. J. Use of detergent in the analysis of fibrous feed. I. A rapid method for detemination of fiber and lignin. Journal of the Association of Official Agricultural Chemists, v. 46, p. 829-835, 1963.

[23] WRIGHT, R.S., ANDERSON, J.W., BRIDGES, S.R. propionate inhibits hepatic lipid synthesis, proceeding of the society for experimental biology and medicine, v. 190, p. 26-29, 1990. 